\title{
A new approach for fuel injection into a solar receiver/ reactor: Numerical and experimental investigation
}

\author{
M Helal Uddin ${ }^{1}$, Nesrin Ozalp $\left.(\bowtie)\right)^{1}$, Jens Heylen ${ }^{2}$, Cedric Ophoff ${ }^{2}$ \\ 1 Mechanical and Industrial Engineering Department, University of Minnesota Duluth, Duluth, MN 55812-3042, USA \\ 2 Mechanical Engineering Department, 3001 Leuven, Belgium
}

(C) Higher Education Press and Springer-Verlag GmbH Germany, part of Springer Nature 2018

\begin{abstract}
An innovative and efficient design of solar receivers/reactors can enhance the production of clean fuels via concentrated solar energy. This study presents a new jet-type burner nozzle for gaseous feedstock injection into a cavity solar receiver inspired from the combustion technology. The nozzle design was adapted from a combustion burner and successfully implemented into a solar receiver and studied the influence of the nozzle design on the fluid mixing and temperature distribution inside the solar receiver using a $7 \mathrm{~kW}$ solar simulator and nitrogen as working fluid. Finally, a thorough computational fluid dynamics (CFD) analysis was performed and validated against the experimental results. The CFD results showed a variation of the gas flow pattern and gas mixing after the burner nozzle adaptation, which resulted an intense effect on the heat transfer inside the solar receiver.
\end{abstract}

Keywords solar reactor, nozzle, CFD, heat transfer, mixing and recirculation

\section{Introduction}

Conventional thermochemical processes use fossil energy sources for producing fuels and commodities. These thermochemical processes are highly energy and carbon intensive [1]. Alternatively, solar thermochemical processes have high potential for the production of clean fuels and commodities via significantly less carbon footprint. Adaptation of conventional process technologies and reactor design are necessary to realize efficient solar thermochemical processes. As a first step, design of an efficient solar reactor lays the foundation of understanding the process dynamics and reactor performance of solar

Received March 12, 2018; accepted July 15, 2018

E-mail: nozalp@d.umn.edu thermal processes. There have been a number of remarkable solar reactors designed and fabricated in the field of solar thermochemistry $[2,3]$. A cyclone-type solar reactor was first developed at the Weizmann Institute of Science for the decomposition of methane [4]. Inspired by the natural tornado phenomenon, the fluid is injected around the axis of the solar reactor to obtain a good mixing that ultimately ensures better heat transfer and to keep the reactor walls clean from particle deposition. Ozalp and Kanjirakat [5] analyzed different aspect of that cyclonetype solar reactor through validated numerical simulations. The results of ref. [5] showed the mixing of fuels via cyclone driven flow dynamics yielding enhanced heat transfer inside the cavity receiver when fuels are introduced at an angle rather than radial direction. The findings provided in ref. [5] suggested a $30^{\circ}$ inlet angle to ensure maximum tangential velocity with swirling flow pattern.

Hirsch and Steinfeld [6] developed a cavity vortex-flow solar reactor to effectively capture the incident solar radiation for methane cracking process to produce hydrogen. The authors reported that the vortex flow created by the helical grooves enhances the heat transfer. This experimental observation was explained through numerical study by Ozalp and JayaKrishna [7] concluding that the reactor wall carving enhances gas mixing and improves methane conversion inside the cavity solar reactor. Abanades et al. [8] designed a packed bed tubular solar reactor to study decomposition of methane for hydrogen production. The reactor design was made to achieve longer residence time of the fluid inside the solar reactor to improve contact between the fluid phase and the solid catalysts for efficient heat transfer. Kodama et al. [9] studied the gasification of coal in a fluidized-bed solar thermal reactor, utilizing the excellent mixing and heat transfer characteristics of fluidization. With this in mind, Kodama et al. [10] proposed and developed a novel design concept for chemical receiver-reactors using an internally 
circulating fluidized bed of reacting particles. Roeb et al. [11] proposed a novel solar reactor configuration similar to that encountered in automobile exhaust catalytic converters. They designed a ferrite-coated SiC-based honeycombs solar reactor to enhance the contact between reactants to perform the water splitting reaction. Muhich et al. [12] provide a comprehensive review on this type of reactor design.

While many of these studies focus on different types of solar receivers/reactors concepts to maximize the use of incoming solar energy through efficient mixing, other design constraints need to be addressed additionally. Bertocchi et al. [13] pointed out the structural and material limitations that needs to consider in protection of the solar receiver's wall from the high temperature exposure under concentrated solar radiation. In a recent review, Kodama et al. [14] also highlighted the severe thermal shocks that often take place under intermittent concentrated solar radiation. It is important to note that an uneven distribution of the concentrated solar radiation inside the cavity receiver creates localized hotspot, generating temperatures beyond the structural and material limitation. Levêque et al. [15] described the existence of such hotspot on planes behind the focal plane (i.e., potentially on a cavity's wall) for a multi-light source solar simulator. More recently, Ophoff et al. [16] summarized the uneven flux distribution inside a cylindrical cavity receiver through a series of numerical simulations for a range of aperture sizes. The authors observed an increasing flux density with the axial and radial direction, respectively, for any aperture diameter larger than $5 \mathrm{~mm}$, arguably shifting the radiative hotspot from the front of the reactor to the back. Their analysis also pointed out that the back plate, highlighting the susceptibility of hotspot, absorbed more than $55 \%$ of the incident energy. Costandy et al. [17] reported analogous observation indicating a large temperature gradient along the longitudinal direction of the solar receiver per computational fluid dynamics (CFD) simulation. To understand the presence of carbon black particles as the radiation absorbing media in air or nitrogen as the working fluid, Klein et al. [18] reported a temperature gradient along the length of their solar reactor, a low temperature at the front followed by a high temperature at the exit. Bertocchi et al. [13] also reported similar observation for the experimental evaluation of their solar particle receiver. Equally, Rodat et al. [19] encountered a temperature gradient in their pilotscale solar reactor operated at the $1 \mathrm{MW}$ solar furnace of CNRS for methane decomposition study. For the same solar furnace at CNRS, Koepf et al. [20] observed the temperature gradient along the length of the solar reactor for thermal reduction of $\mathrm{ZnO}$ as part of a two-step water and $\mathrm{CO}_{2}$ splitting cycle.

This literature survey highlights the shortcomings of an efficient solar reactor that can overcome the challenges of: (1) Efficient fluid mixing and (2) Uniform flux/temperature distribution to avoid high temperature gradient inside the solar reactor. With proper design and fabrication of the solar reactor, it is possible to maintain a uniform temperature distribution and to avoid the hotspot inside a solar receiver. Given the prospect and importance of solar thermochemical processes, more innovative design of solar receivers/reactors is deemed necessary for successful solar thermal applications. This study demonstrates a new design perspective of a solar receiver/reactor by adapting combustion process technology. The main motivation thrives from the fact that solar receivers/reactors basically function like combustors where the only difference is the source of heat for endothermic reactions. Since combustion technologies are well studied, the knowledge generated from those studies can provide foundation to assist in advancing solar thermochemical technology.

For combustors, the effective momentum of the fuel is enhanced by introducing some of the air through the burner nozzle as primary air [21]. Such a configuration allows the momentum of the combined fuel and primary air streams to dominate over the rest of the combustion air. The primary goal is to enhance fuel mixing as it controls the efficiency of the combustion process [22]. In most combustion systems, the mixing is slow while the other steps are fast [21]. For one type of combustion, namely jet combustion, is an effective way to increase the fuel mixing. The most important feature of the jet combustion is its capability to create a swirling flow inside the combustion chamber. Swirling flow pattern compromises different turbulent flow pattern, facilitating intense mixing between the phases under studied, enhancing heat and mass transfer inside a reaction chamber. Additionally, jet flow delivers high momentum and good recirculation of the fluid inside the chamber [21]. Thus, by adapting the jet combustion idea into a solar receiver/reactor, it is largely expected to achieve a homogenous temperature distribution by assuming a uniform mixing of the fluid inside the solar receiver/ reactor.

To understand the elementary reactions describing the nucleation, growth, and reoxidation of $\mathrm{Zn}$ in a cavity solar reactor, Koepf et al. [20] used cavity jets to supply a sweeping argon flow along the reaction surface of the cavity annular. However, they did not inject the sweeping gas for mixing or enhancing heat transfer inside the cavity, rather any turbulence disturbed their process. Previously, the Solar Thermal Technology Laboratory (STTL) at the KU Leuven designed and tested a cavity solar receiver/ reactor where three tangential inlet ports next to the quartz aperture were used for the gaseous feedstock injection to study non-reactive fluid mixing and heat transfer inside the solar receiver [23]. In present study, we explore the applicability of the jet combustion concept into that solar receiver for injecting nitrogen. A jet-type nozzle was designed for the gas injection into the solar receiver, analogous to the jet combustion process. The nozzle design was adapted from a jet combustion burner concept developed by Vanierschot [24]. The influence of the gas 
mixing and temperature distribution inside the solar receiver due to the nozzle inclusion are investigated both experimentally and numerically.

\section{Nozzle design}

The original nozzle design is based upon a concept developed by Vanierschot [24] for a burner, which was successful per experimental observation at the KU Leuven Mechanical Engineering Department and was available for detailed inspection during this research. The most important feature of the nozzle is its capability to create a swirling flow inside the combustion chamber providing intense mixing of the reactant gases. It was expected that adaption of this technology into solar receiver/reactor would yield similar flow pattern and homogenous temperature distribution. A symmetric cross section view of the jet-type nozzle developed by Vanierschot [24] is shown in Fig. 1.

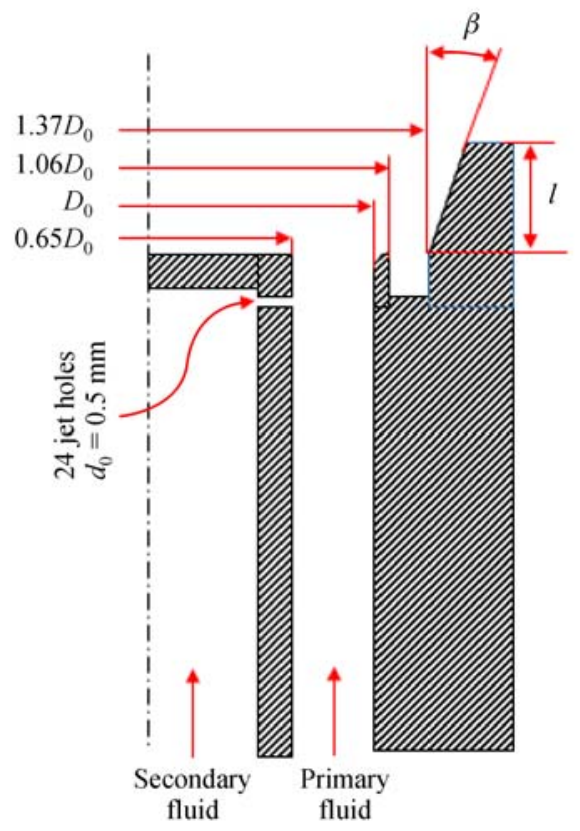

Fig. 1 A simplified schematic of cross section of the nozzle [24]

To make sure that the nozzle is compatible with the solar receiver, a few adjustments had to be made [25]. To begin with, a flange was attached to the outer nozzle. Then a new method of centralizing the inner rod and the outer part had to be found. To centralize the inner rod with the outer nozzle, the back of the outer part was made completely closed except for a hole where the inner rod could fit through. The new inner rod has 16 holes with a diameter of $1 \mathrm{~mm}$ equally spaced at $5 \mathrm{~mm}$ from the end of the rod. To provide the annular channel for air, two injections were symmetrical placed on the sides. The last change on the outer nozzle was the dimensions. The solar reactor adopted nozzle design is composed in two parts - an inner hollow rod and an outer annulus. The outer diameter of the annular channel, $D_{0}$, is the characteristic dimension of the nozzle. The dimensions used are $D_{0}=29.3 \mathrm{~mm}, l=11.9 \mathrm{~mm}$, and $\beta=20^{\circ}$. As shown in Fig. 1, two fluid streams - a primary and a secondary - enters through the nozzle. Since the outer part is a stepped conical with a slot, the sub-pressure of the corner recirculation zone behind the sudden expansion increases. This allows to control the flow pattern inside the solar receiver by adjusting the radial injection through the small opening jets. Figure 2 demonstrates the solar receiver adapted nozzle design.

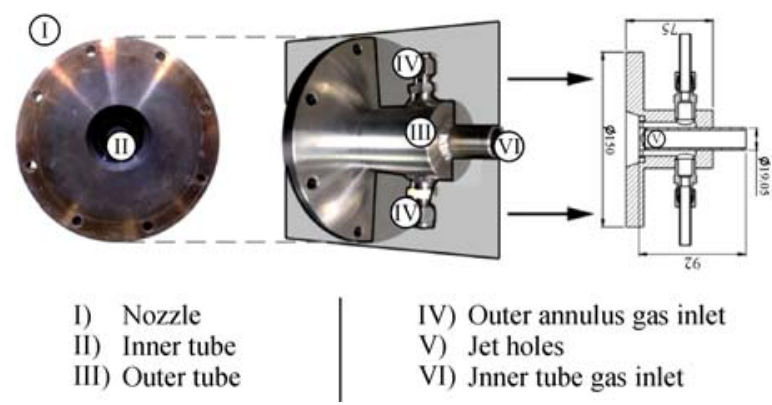

Fig. 2 Different view of the nozzle designed for solar reactor: (left) front, (middle) isometric, and (right) cross section of the nozzle [25]. All dimensions are shown in $\mathrm{mm}$

\section{Experimental setup}

The adapted nozzle was experimentally tested with the solar receiver at the STTL of KU Leuven using a $7 \mathrm{~kW}$ high flux solar simulator (Proyecson Xenolux H). It was mounted to a cavity solar receiver which was placed at the focal point of the solar simulator. The cylindrical solar receiver was made of stainless steel with length, inner radius, and outer radius of 200, 60 and $75 \mathrm{~mm}$, respectively. Concentrated solar flux entered the cavity through a $60 \mathrm{~mm}$ aperture mounted at the front flange. Flux levels of this solar simulator were adjusted through the input current. The cavity and wall temperatures were measured by $11 \mathrm{~K}$-type thermocouples (Omega M12KIN) implanted symmetrically around the solar receiver. Six horizontally inserted thermocouples, two at each section of the reactor (front, middle, and back), measured the gas temperature inside solar receiver. Four vertically placed thermocouples, two in the middle and two in the back, recorded the wall temperatures. The time series data of temperature was logged using a NI CompactRio and LabVIEW. Temperature distribution inside the cavity receiver was studied for two experimental configurations. In the first configuration, nitrogen entered the cavity through three tangential inlets (ID: $9.25 \mathrm{~mm}$, each) at a 
total flowrate of $0.6 \mathrm{~m}^{3} \cdot \mathrm{h}^{-1}$ and left through a $10.46 \mathrm{~mm}$ radius exit port at the back plate. In second configuration, the back plate was replaced with the jet-type nozzle. Nitrogen at 0.52 and $0.08 \mathrm{~m}^{3} \cdot \mathrm{h}^{-1}$ flowed through the annulus and inner tube, respectively. The gas exit from the cavity through the three ports used as inlet for the first configuration. For all experiments, the solar simulator input current was 155 A. Figure 3 shows the overview of experimental setup.

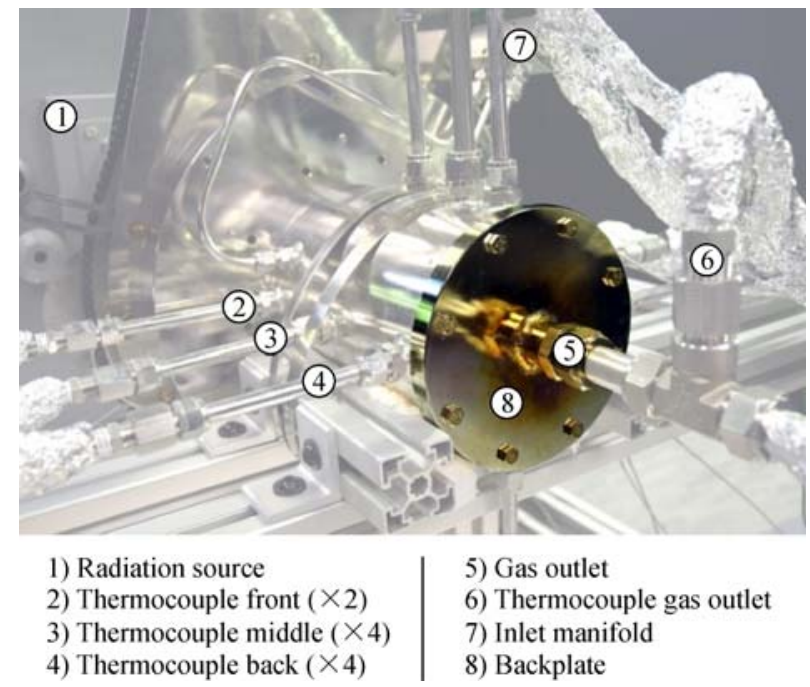

Fig. 3 Experimental setup at the STTL of KU Leuven

\section{Numerical analysis}

\subsection{Model and solver}

The solar receiver with the new nozzle was numerically simulated using commercial software ANSYS FLUENT 18.0. The flow field of the model was divided into a finite number of small volume elements, where the global and local balances of continuity, momentum, and energy were conserved for a predefined convergence threshold $\left(10^{-6}\right)$. Pressure-based SIMPLE algorithm was used for pressuremomentum coupling. PRESTO scheme was applied for face pressure interpolation. Second order upwinding method was used for the interpolation of all other variables. The realizable $k-\varepsilon$ turbulence model was chosen for turbulence modeling since it provides improved predictions for the spreading rate of jets. The governing equations that were solved numerically are given in Eqs. (1-7). The equation for conservation of mass or continuity for an incompressible flow under steady state:

$$
\nabla \cdot(\rho \vec{u})=0 .
$$

The steady state equation for conservation of momentum in an inertial reference frame, neglecting the fluid gravitational body force: $\nabla \cdot(\rho \vec{u} \vec{u})=-\nabla P+\nabla \cdot \mu_{\mathrm{eff}}\left[\left(\nabla \vec{u}+\nabla \vec{u}^{\mathrm{T}}\right)-\frac{2}{3} \nabla \cdot \vec{u} \lambda\right]$.

The equation for energy conservation for a non-reactive and radiative heat source at steady state:

$$
\begin{gathered}
\nabla \cdot[\vec{u}(\rho E+P)]=\nabla \cdot\left(k_{\mathrm{eff}} \nabla T\right. \\
+\vec{u} \mu_{\mathrm{eff}}\left[\left(\nabla \vec{u}+\nabla \vec{u}^{\mathrm{T}}\right)-\frac{2}{3} \nabla \cdot \vec{u} \lambda\right]+Q_{\mathrm{rad}}
\end{gathered}
$$

It should be noted that the second term on the right-hand side of the energy equation represent the viscus heating, which is neglected for the gas phase. The realizable transport equations for the turbulence kinetic energy for incompressible flow with strong streamline curvature, vortices, and rotation at steady state:

$$
\nabla \cdot(\rho k \vec{u})=\nabla \cdot\left(\mu+\frac{\mu_{\mathrm{t}}}{\sigma_{\mathrm{k}}}\right) \nabla k+G_{\mathrm{k}}+G_{\mathrm{b}}-\rho \varepsilon .
$$

The model transport equations for the turbulence kinetic energy dissipation rate for incompressible flow with strong streamline curvature, vortices, and rotation at steady state:

$$
\begin{gathered}
\nabla \cdot(\rho \varepsilon \vec{u})=\nabla \cdot\left(\mu+\frac{\mu_{\mathrm{t}}}{\sigma_{\varepsilon}}\right) \nabla \varepsilon+C_{1} \gamma \rho \varepsilon-C_{2} \rho \frac{\varepsilon^{2}}{k+\sqrt{v \varepsilon}} \\
+C_{1 \varepsilon} C_{3 \varepsilon} G_{\mathrm{b}} \frac{\varepsilon}{k}
\end{gathered}
$$

where

$$
\begin{gathered}
\mu_{\mathrm{t}}=C_{\mu} \rho \frac{k^{2}}{\varepsilon}, \\
C_{\mu}=\frac{1}{A_{0}+A_{\mathrm{s}} \frac{k U^{*}}{\varepsilon}}, \\
U^{*}=\sqrt{\gamma_{i, j} \gamma_{i, j}+\tilde{\Omega}_{i, j} \tilde{\Omega}_{i, j}}, \\
A_{\mathrm{s}}=\sqrt{6} \cos \phi, \\
=\frac{1}{3} \cos ^{-1}(\sqrt{6} W), \\
W=\frac{1}{\tilde{\gamma}^{3}} \gamma_{i, j} \gamma_{j, k} \gamma_{k, i}, \\
\tilde{\gamma}=\sqrt{\gamma_{i, j} \gamma_{i, j}},
\end{gathered}
$$




$$
\begin{gathered}
C_{1}=\max \left[0.43, \frac{\eta}{\eta+5}\right], \eta=\gamma \frac{k}{\varepsilon}, \gamma=\sqrt{2 \gamma_{i, j} \gamma_{i, j}}, \\
k_{\mathrm{eff}}=k+\frac{c_{\mathrm{p}} \mu_{\mathrm{t}}}{P r_{\mathrm{t}}} .
\end{gathered}
$$

Detailed description and explanation of these equations can be found in [26].

The incoming solar irradiation into the solar receiver was modeled using discrete ordinate (DO) method. The DO model solves the radiative transfer equation for a finite number of discrete solid angles for the spectral intensity $I(\vec{r}, \vec{s})$ at position $\vec{r}$ in the direction $\vec{s}$ as follows:

$$
\begin{aligned}
\frac{\mathrm{d} I(\vec{r}, \vec{s})}{\mathrm{d} s}= & \frac{a n^{2} \sigma}{\pi} T^{4}-(\alpha+\beta) I(\vec{r}, \vec{s}) \\
& +\int_{0}^{4 \pi} \beta I\left(\vec{r}, \vec{s}^{\prime}\right) \frac{\hat{\phi}\left(\vec{s}, \overrightarrow{s^{\prime}}\right)}{4 \pi} \mathrm{d} \Omega^{\prime} .
\end{aligned}
$$

\subsection{Boundary conditions}

The solar receiver walls were modeled as no-slip boundary. Dirichlet boundary, i.e., uniform inlet gas $\left(\mathrm{N}_{2}\right)$ velocity, was employed at the inlets. Pressure boundary condition was applied at the exit of the cavity reactor.

Figure 4 shows the experimentally measured incident radiation flux of the $7 \mathrm{~kW}$ solar simulator for an input current of $155 \mathrm{~A}$ where a peak flux of $3948 \mathrm{~kW} \cdot \mathrm{m}^{-2}$ was observed at its focal point. The flux data showed symmetric character as concentric contour of circles. An in-house algorithm was used to find the flux values for a specified diameter of the concentric circle. Accordingly, the smallest circle was identified as a diameter of $2 \mathrm{~mm}$ and a peak flux of $3948 \mathrm{~kW} \cdot \mathrm{m}^{-2}$. This flux map was taken as the flux intercepted by the solar receiver aperture. The solar receiver's quartz window (3.1 mm thickness) was modeled as a semi-transparent boundary. Detailed description of the semi-transparent boundary can be found in ref. [26]. The inner walls of the cavity receiver were modeled as opaque walls.

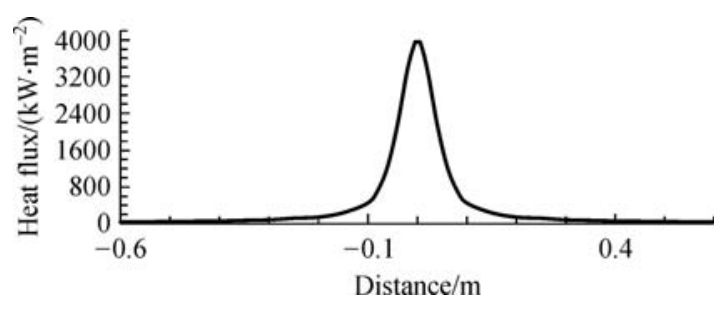

Fig. 4 Experimentally measured heat flux of the $7 \mathrm{~kW}$ solar simulator

The solar receiver wall had a thickness of $15 \mathrm{~mm}$ and an outer layer insulation of $60 \mathrm{~mm}$ (Cerachem Blanket 104-9EURO). Heat conduction through these wall layers was modeled applying shell conduction method with variable thermal properties. Heat transfer from the outer boundary layer of the insulation was modeled as combined radiation and convection thermal boundary. A summary of physical properties used in simulations is given in Table 1.

\subsection{Geometry and discretization}

Three-dimensional flow geometries, analogous to experimental setup, were created in SolidWorks ${ }^{\circledR}$ for CFD analysis. Figure 5 shows the 3-D flow geometry of the solar receiver with and without nozzle for comparison.

These geometries were used to generate non-uniform unstructured tetrahedral mesh for the numerical study. A grid independent study was performed prior to any CFD data analysis, as it is essential to obtain a numerical solution that does not vary with the number of computational cells. Tables 2 and 3 show the results of the grid independence tests. Average gas velocity and dimensionless average wall spacing, $y^{+}$, were used as standard to seek grid independent numerical solution. Since the flow field inside the solar receiver was turbulent, the dimensionless turbulent length was supposed to satisfy $0 \leqslant y^{+} \leqslant 1$ [26].

As shown in Tables 2 and 3 , the gas velocity and $y^{+}$ values on the wall decreased with increased number of tetrahedral cells. However, a 2.64 folds increase in computational cells from 646716 to 1707881 resulted in less significant changes in the average velocity and $y^{+}$ values. The maximum skewness in both cases was within acceptable threshold for both cases [30]. Therefore, acknowledging this small discrepancy and avoiding the added computational cost for very fine grid, a grid cell number 646716 was chosen as grid independent solution for further analysis of the solar receiver geometry without nozzle. Similar argument was also made for the solar receiver geometry with nozzle, and, a grid cell number of 1090884 was chosen for the rest of the analysis.

\section{Results and discussions}

\subsection{Experimental results without nozzle}

Initial experiments were performed without the nozzle (Fig. 6(a)) and it was referred as a reference case for the rest of the discussion. Figure 6(b) shows the change of temperature distribution measured by different thermocouples where the front (G0 and G1) section had two, and middle (G2, G3, W1, W2) and back (G4, G5, W3, W4) sections had four thermocouples each.

Temperature measurements by two thermocouples were averaged to obtain distribution profile at each section. As seen in Fig. 6(b), the average temperature at each section increases during transient period. When the overall heat exchange between the gas and receiver walls reached to a thermal equilibrium, the steady state was achieved. It was 
Table 1 Physical properties of the simulation parameters

\begin{tabular}{|c|c|c|}
\hline Physical properties & Correlations & Ref. \\
\hline Density $/\left(\mathrm{kg} \cdot \mathrm{m}^{-3}\right)$ & $\begin{array}{c}\rho_{\mathrm{g}}(T)=\frac{P M}{\mathrm{R} T} \\
\rho_{\mathrm{ss}}=7970^{*}, \rho_{\text {ins }}=128^{*}, \rho_{\text {qua }}=2214^{*}\end{array}$ & Manufacturer supplied \\
\hline \multirow[t]{2}{*}{ Specific heat $/\left(\mathrm{J} \cdot \mathrm{kg}^{-1} \cdot \mathrm{K}^{-1}\right)$} & $\begin{array}{c}C_{p_{\mathrm{g}}}(T)=947.51+0.26667 T-5 \times 10^{-5} T^{2} \\
C_{\mathrm{pss}}(T)=349.145+0.469 T-4.007 \times 10^{-4} T^{2}+1.34 \times 10^{-7} T^{3}\end{array}$ & {$[27,28]$} \\
\hline & $C_{\mathrm{p}_{\text {ins }}}=1130^{*}, C_{\mathrm{p}_{\text {qua }}}=700^{*}$ & Manufacturer supplied \\
\hline Viscosity $/\left(\mathrm{kg} \cdot \mathrm{m}^{-1} \cdot \mathrm{s}^{-1}\right)$ & $\mu_{\mathrm{g}}(T)=4 \times 10^{-5}+5 \times 10^{-7} T-10^{-10} T^{2}$ & {$[29]$} \\
\hline \multirow{3}{*}{ Thermal conductivity $/\left(\mathrm{W} \cdot \mathrm{m}^{-1} \cdot \mathrm{K}^{-1}\right)$} & $k_{\mathrm{g}}(T)=0.0146+5 \times 10^{-5} T$ & {$[27,28]$} \\
\hline & $k_{\mathrm{ss}}(T)^{*}=11.133+0.013 T$ & Manufacturer supplied \\
\hline & $\begin{array}{c}k_{\text {ins }}(T)^{*}=0.0504-10^{-4} T+3 \times 10^{-7} T^{2} \\
k_{\text {qua }}=1.4^{*}\end{array}$ & \\
\hline
\end{tabular}

* Indicates manufacturer provided data

observed that there was a small temperature difference between the gas and reactor walls. For example, the average temperature measured by horizontally placed thermocouples $\mathrm{G} 2$ and $\mathrm{G} 3$ differs only by $10 \mathrm{~K}$ from the temperature measured by vertically placed thermocouples $\mathrm{W} 1$ and W2. This indicates that most of the heat is absorbed by the receiver walls. However, Figure 6 shows large temperature gradient along the length of the solar receiver. The difference between the mean temperature measured by thermocouples at the back (G4 and G5) and at the front (G0 and G1) is about $200 \mathrm{~K}$. Similarly, the middle (G2, G3 and W1, W2) and the back (G4, G5 and W3, W4) thermocouples shows about $115 \mathrm{~K}$ difference. This wide temperature difference resulted from the uneven radiation flux distribution inside the solar receiver where the majority of beams hit the back plate. The back plate exchanges radiation with the sidewalls via emission and reflection. Because the gas phase inside the solar receiver is transparent to receiver walls are the main heat transfer domain. Direct exposure to beams as well as emitted and reflected flux from the sidewalls facilitates high temperature zone at the back plate, which is called hotspot. For the feedstock entry, three tangential ports were used to inject nitrogen to create swirl motion of the gas inside the solar receiver to enhance the heat transfer. However, such enhancement diminishes as swirl decays towards exit of the receiver resulting a near-stagnant high temperature zone at the back.

\subsection{Experiment with jet-type nozzle}

The jet-type nozzle described in Section 2 was manufactured at the STTL of KU Leuven and tested with the solar receiver by replacing the back plate as highlighted in Fig. 3. Nitrogen flow was reversed and entered from the back and exited from the front as shown in Fig. 7(a). The average temperature distribution is shown in Fig. 7(b).
Like the reference case without the nozzle, a temperature gradient was observed along the length of the solar receiver.

In this case, the temperature difference at the front and the back of solar receiver was about $159 \mathrm{~K}$, which was $41 \mathrm{~K}$ less than the reference case. Such reduction in temperature difference is promising but it still lacks in maintaining a uniform temperature distribution and the hotspot issue remains at the back plate. Thus, design of the jet-type nozzle was needed to be improved which was numerically explored as described in Section 5.4.

\subsection{Numerical results}

\subsubsection{Validation}

Numerical analysis was performed to obtain more insights about the flow behavior and heat transfer inside the solar receiver. First, the numerical method was validated by comparing the average gas temperatures at three locations with the experimental results. Table 4 shows the summary of the validation results.

As it is seen in Table 4, the simulation results overpredicted the front and the middle sections' temperature by maximum of $4.7 \%$ for both cases. Simulation results under-predicted the back-section temperature by maximum of $4.7 \%$. The cumulative average gas temperature varies only by $2 \%$. There are number of reasons for this discrepancy. First, numerical simulation assumed a steady state thermal equilibrium but the temperature was still rising at the end of the experiment (Figs. 6(b), 7(b)). Because, true thermal inertia and steady state were never achieved experimentally. Second, the difference between the theoretical and effective physical properties of materials used in simulation are likely to contribute an error as well [31]. Finally, the thermocouples that 
(a)

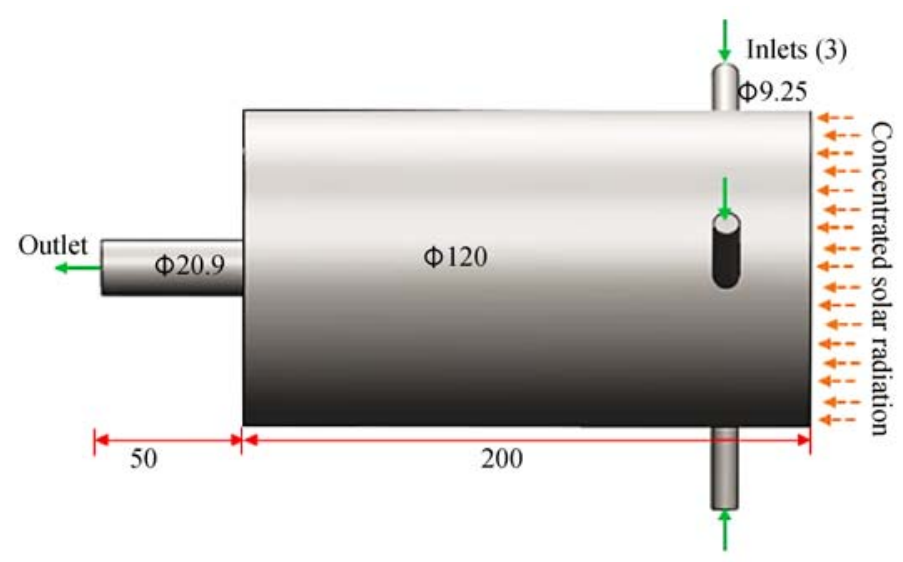

(b)
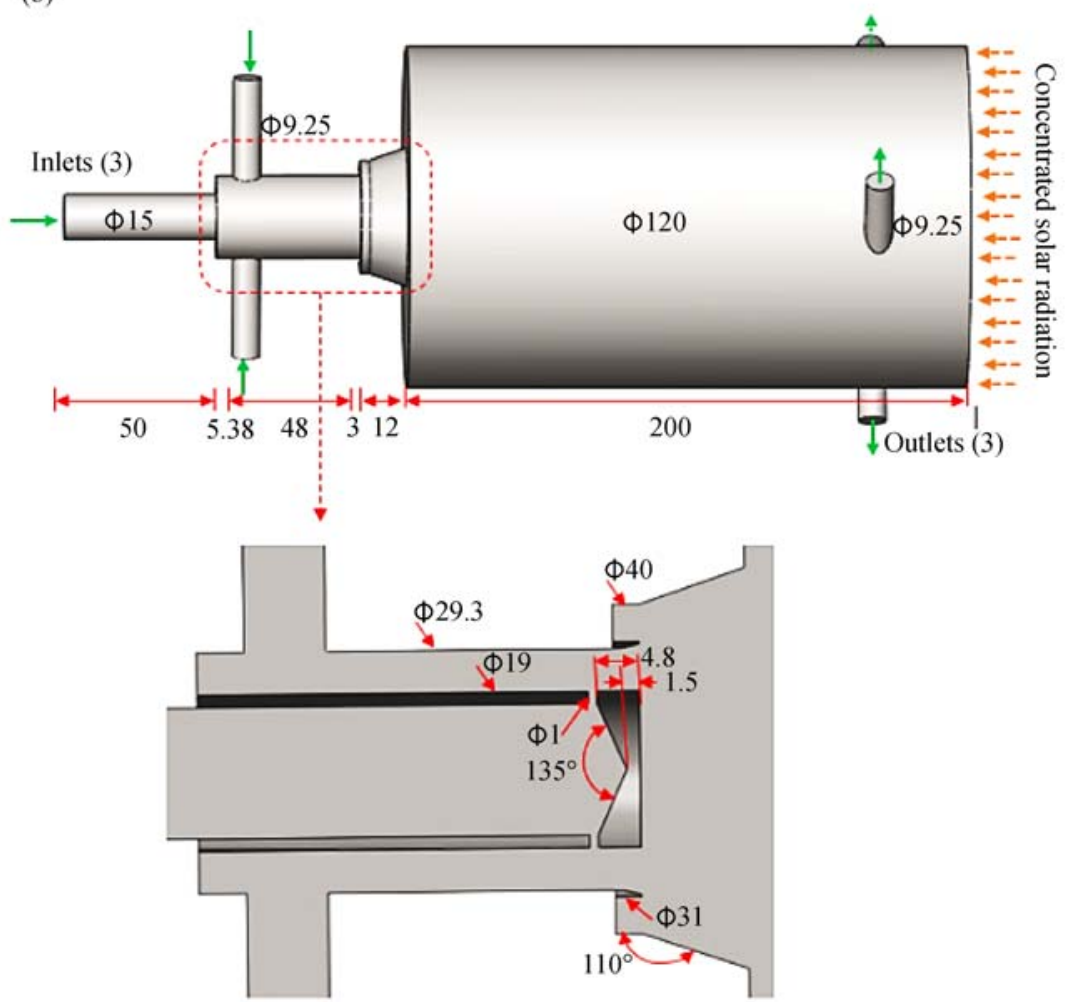

Fig. 5 Receiver geometry: (a) without nozzle and (b) with jet-type nozzle. All dimensions except angles are shown in mm

Table 2 Grid independent test results without the nozzle

\begin{tabular}{|c|c|c|c|c|}
\hline Simulation number & $\begin{array}{l}\text { Grid size (number of tetrahedral } \\
\text { cells) }\end{array}$ & Maximum skewness & $\begin{array}{l}\text { Area-weighted average velocity at exit/(cm } \\
\left.\qquad \cdot \mathrm{s}^{-1}\right)\end{array}$ & Average $y^{+}$on the wall \\
\hline 1 & 314415 & 0.84 & 53.37 & 1.67 \\
\hline 2 & 411357 & 0.83 & 53.13 & 1.32 \\
\hline 3 & 646716 & 0.84 & 52.52 & 0.99 \\
\hline 4 & 1707881 & 0.85 & 52.11 & 0.44 \\
\hline
\end{tabular}

were exposed to measure gas temperature during experiment might experience radiation interference by being exposed to some of the beams directly coming from the simulator. Despite these limitations, the numerical results showed an appreciable agreement with the experimental results. 
Table 3 Grid independent test results with nozzle

\begin{tabular}{lcccc}
\hline Simulation number & $\begin{array}{c}\text { Grid size (number of tetrahedral } \\
\text { cells) }\end{array}$ & Maximum skewness & $\begin{array}{c}\text { Area-weighted average exit velocity/(cm } \\
\left.\cdot \mathrm{s}^{-1}\right)\end{array}$ & 1.25 \\
\hline 1 & 798587 & 0.84 & 105.53 & 0.95 \\
2 & 1090884 & 0.84 & 105.06 & 0.76 \\
3 & 1274733 & 0.85 & 104.86 & 0.76 \\
\hline
\end{tabular}

(a)

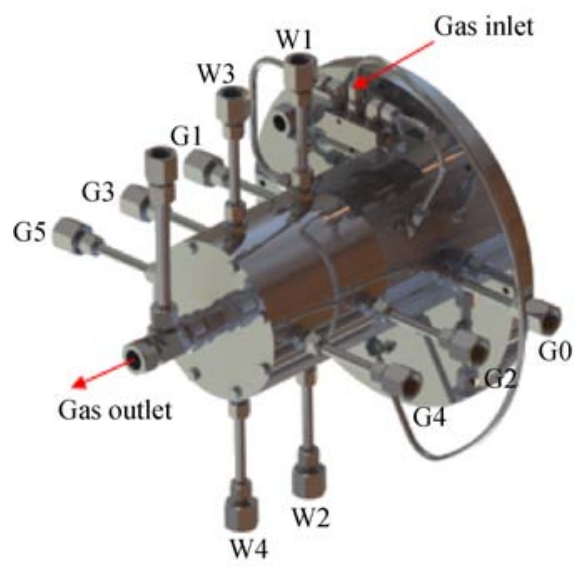

(b)

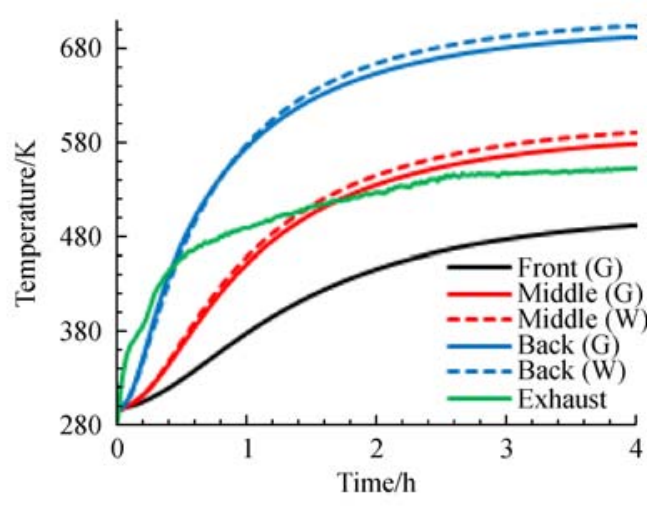

Fig. 6 Solar receiver without nozzle: (a) thermocouple positions and (b) temperature measurement profiles for an inlet $\mathrm{N}_{2}$ flow of $0.6 \mathrm{~m}^{3} \cdot \mathrm{h}^{-1}$ where $\mathrm{G}, \mathrm{W}$, and $\mathrm{E}$ indicate the gas, wall, and exhaust

(a)

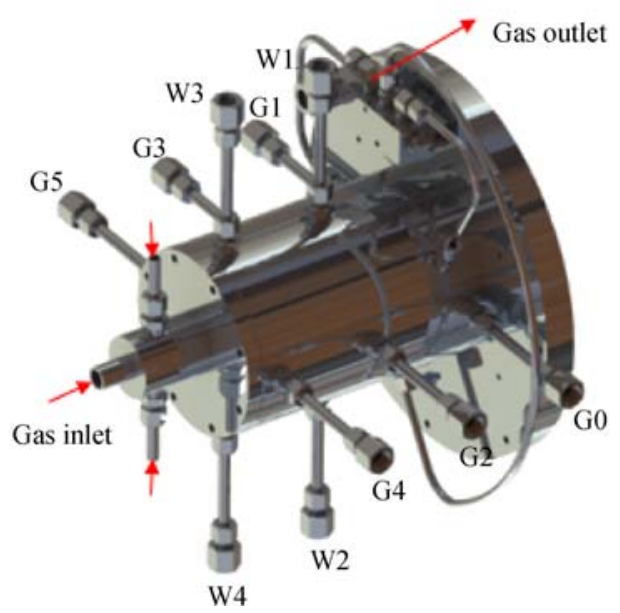

(b)

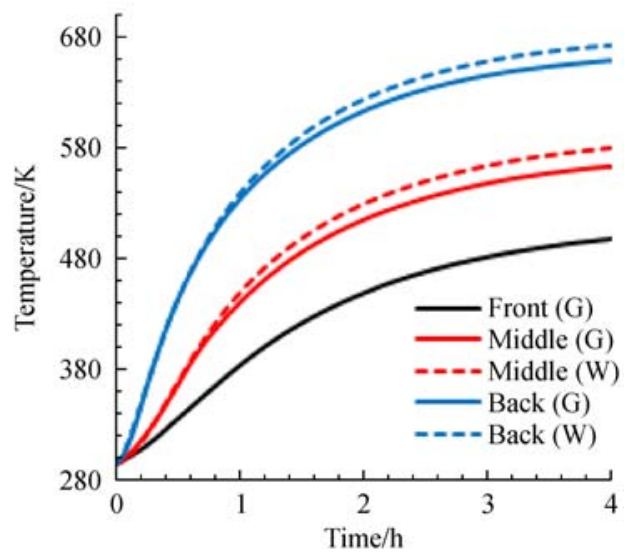

Fig. 7 Solar receiver with nozzle: (a) thermocouples position, and (b) measured temperature profile for the inlet $\mathrm{N}_{2}$ flow of $0.6 \mathrm{~m}^{3} \cdot \mathrm{h}^{-1}$ and the input current of $155 \mathrm{~A}$

\subsubsection{Flow and temperature profile}

Since the numerical analysis was in good agreement with the experimental results, the same numerical analysis was used to further study the flow and temperature characteristics inside the solar receiver. Figure 8 shows the gas flow behavior inside the solar receiver. For the reference case shown in Fig. 8(a), the gas entered the solar receiver at $30^{\circ}$ inlet angle which facilitated cyclone type-flow with swirling inside the receiver.

The inlet angles were defined with respect to the tangential direction of the receiver. It is seen in Fig. 8(a) that the swirl strength was gradually reduced towards the exit indicating larger shear loss of the fluid with the 
Table 4 Comparison of simulation results with experimental results

\begin{tabular}{|c|c|c|c|c|c|c|}
\hline \multirow[b]{2}{*}{ Location } & \multicolumn{2}{|c|}{ Temperature/K } & \multirow[b]{2}{*}{ Error $/ \%$} & \multicolumn{2}{|c|}{ Temperature/K } & \multirow[b]{2}{*}{ Error/\% } \\
\hline & $\begin{array}{c}\text { Experiment without } \\
\text { nozzle }\end{array}$ & $\begin{array}{c}\text { Simulation without } \\
\text { nozzle }\end{array}$ & & $\begin{array}{l}\text { Experiment with } \\
\text { jet-type nozzle }\end{array}$ & $\begin{array}{l}\text { Simulation with } \\
\text { jet-type nozzle }\end{array}$ & \\
\hline Front & 494 & 500 & -1.2 & 497 & 518 & -4.2 \\
\hline Middle & 580 & 608 & -4.7 & 564 & 590 & -4.6 \\
\hline Back & 694 & 669 & 3.6 & 663 & 632 & 4.7 \\
\hline Average & 589 & 598 & -1.7 & 573 & 585 & -2.1 \\
\hline
\end{tabular}

(a)

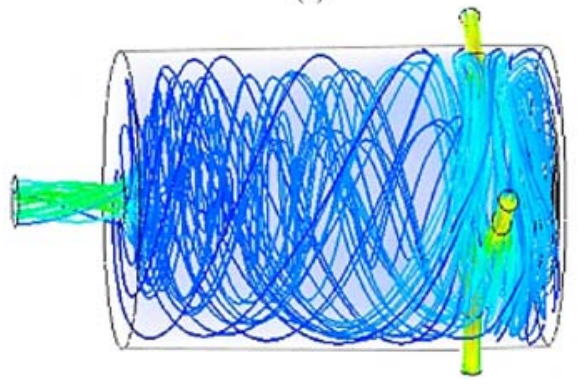

(b)

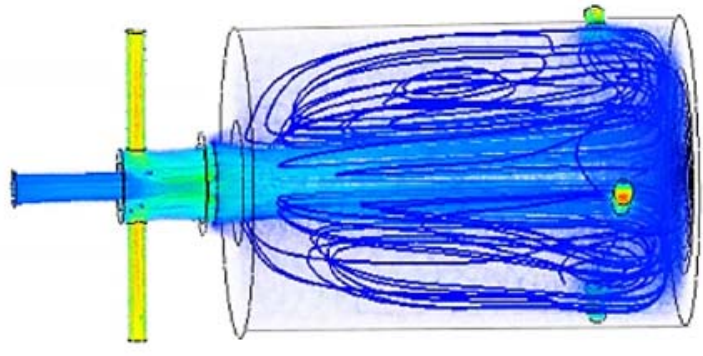

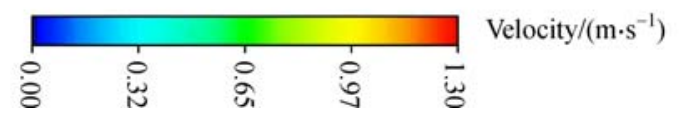

Fig. 8 Gas velocity streamlines in solar receiver: (a) without nozzle, and (b) with nozzle

receiver wall and with the adjacent fluid layers. Figure 8(b) shows the velocity profile with the nozzle attached at the back of the receiver. The gas flow lines are jet-like bundles and there is no visible swirl. The flow proceeds towards the window forming a plug flow at the core due to the high gas velocity at the center. The large shear from the near stagnant gas brings down the velocity and makes it zero at the wall. Due to the low gas movement or circulation next to the entrance, a stagnation zone is likely to form at those locations.

It is seen that the temperature distribution shows symmetric behavior with respect to the axis planes (Fig. 9). In all cases, low temperature at the front continues to increase with the cavity length and reaches to maximum temperature at the back analogous to the experimental observation. The gas temperature near the wall is higher than any location inside the receiver and possesses a maximum temperature at the back plate. This can be explained through the momentum boundary assumption used in the numerical simulation. Under no-slip condition, the rate of conduction is equal to the rate of convection at the gas-solid interface. Since the solar receiver walls absorb most of the radiation heat, the inner wall surfaces achieve high temperature. Thus, the gas layer at the wall also reaches to high temperature as seen in Fig. 9. However, the heat transfer rate starts slowing down away from the wall surfaces as heat is exchanged between gas layers through convection. This results low temperature at the center of the receiver.

The temperature gradient along the receiver length can also be comprehended from the gas flow dynamics. As seen in Fig. 8(a), the tangential flow introduces a swirling characteristic that enhances the convective heat transfer between the gas and the receiver wall []. Thus, the force convection heat transfer is high near the entrance, which helps to lower the wall temperature and to raise the incoming gas temperature quickly []. Nonetheless, the swirl strength and gas velocity decreases with increasing receiver length and reaches to nearly at rest at the back of the receiver. As explained in Section 5.1, the back plate is susceptible to over-heating due to the uneven radiation flux distribution inside the cavity, and that facilitates high temperatures by the stagnant gas as well.

It is seen that the overall gas temperature distribution shows an improvement for the solar receiver with nozzle as shown in Figs. 9(c,d). This can be explained referring to the theoretical flow schematic of a free jet as illustrated in Fig. 10. Fluid from a jet has higher momentum than that of the surrounding fluid which creates internal recirculation to mixing fluid within the jet core []. If the fluid momentum is large enough, it entrains the surrounding fluid and spreads it in the radial direction with downstream. Such fluid entrainment creates an external recirculation and breaks the stagnant dead zone near the entrance and wall regions as 
seen in Fig. 10. The presence (or absence) of recirculation has a great influence on the fluid mixing and subsequent fluid temperature distribution. Lack of such recirculation facilitates temperature gradient as seen in Figs. 9(c,d).

\subsection{Proposed modification to nozzle design}

Per aforementioned discussions in Sections 5.2 and 5.3, the nozzle adaptation did not substantially improve the overall temperature distribution. Therefore, another design concept was explored: (1) to minimize large temperature gradient, (2) to ensure uniform temperature distribution, and (3) to protect the receiver back wall from overheating. The proposed configuration for the nozzle is shown Fig. 11.

This design has eight jets placed symmetrically around the nozzle with an ID $=0.5 \mathrm{~mm}$. The $10^{\circ}$ horizontal angle of the jet outlet is used to divert the gas flow towards the back plate. Such jet-type nozzle was first designed by the VGB Powertec company [28] for their circulating fluidized bed combustors. The pressure drop through the jet causes large disturbance in the flow field which facilitates an intense gas circulation. This circulation provides a good degree of gas phase mixing, enhances heat and mass transfer rates. A rule of thumb from practical application is that a pressure drops of $2500 \mathrm{~Pa}$ or higher at the jet exit will ensure good gas distribution in the chamber [28]. The pressure drop can be calculated from:

$$
\Delta P=\frac{1}{2 K_{\mathrm{d}}^{2}} \rho v_{j}^{2} .
$$

where $K_{\mathrm{d}}=0.8$ for orifice discharge coefficient, $\rho$ is the gas density and $v_{j}$ is the gas exit velocity of the jet. For this modified jet design, the pressure drop across jets, $\Delta P$, is $8021 \mathrm{~Pa}$ with Reynolds number of 3643 for an inlet gas flow of $0.6 \mathrm{~m}^{3} \cdot \mathrm{h}^{-1}$. Thus, this design ensures both large pressure drop and turbulent flow.

\subsubsection{Flow dynamics}

The gas flow dynamics inside the solar receiver was studied numerically for the proposed jet-type nozzle. Based on the grid independent study shown in Table 3, it is observed that grid independent results can be obtained with tetrahedral cells exceeding 1000000 . For this simulation, we used a total number of 1284241 tetrahedral cells. The average value of $y+$ on the wall was 0.54 . Figure 12 shows the gas velocity path lines for the modified nozzle.

The gas flow patterns inside the receiver highlight an intense gas circulation at the back due to large pressure drop across the jet which creates turbulent flow with vigorous gas mixing. However, the intensity of the gas circulation reduces as it moves towards the exit of the solar receiver (Fig. 12(a)). It should be noted that small horizontal angle for the jet outlet ensures the gas impingement on the back plate as seen in Fig. 12(b). It helps to quickly reduce the back section's temperature as the gas enters at low temperature where mixing enhances the heat transfer. The impinging gas velocity, $v_{\mathrm{im}}$, on the back plate can be predicted from the centerline-jet velocity decay law []:

$$
v_{\mathrm{im}}=\frac{B \sqrt{M_{\mathrm{f}}}}{l_{\mathrm{im}}} .
$$

According to [], the constant, $B$, has a value of 7 . For a free jet, momentum flux, $M_{\mathrm{f}}$, is conserved and its value is equal to $A_{j} v_{j}{ }^{2}$, where $A_{j}$ is jet cross-sectional area of the jet hole. $l_{\text {im }}$ is the impinging length from the jet centerline and can be calculated from the geometry. Applying Eq. (9), the gas impinging velocity is about $7.3 \mathrm{~m} \cdot \mathrm{s}^{-1}$ on the back plate. The simulation results also show an impinging velocity that is much closer to the theoretical one as seen in
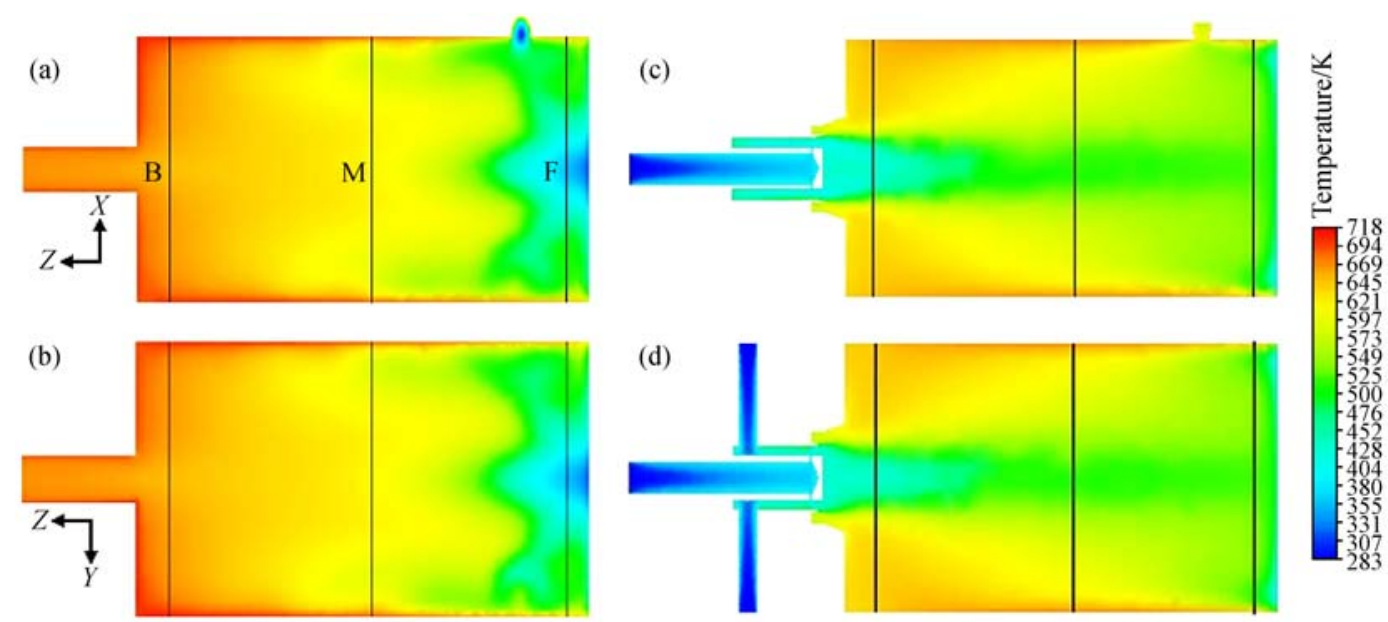

Fig. 9 Contours of static temperature of receiver: ( $a$ and b) without nozzle and (c and d) with nozzle. F, M, and B indicates front-, middle- and back-planes 


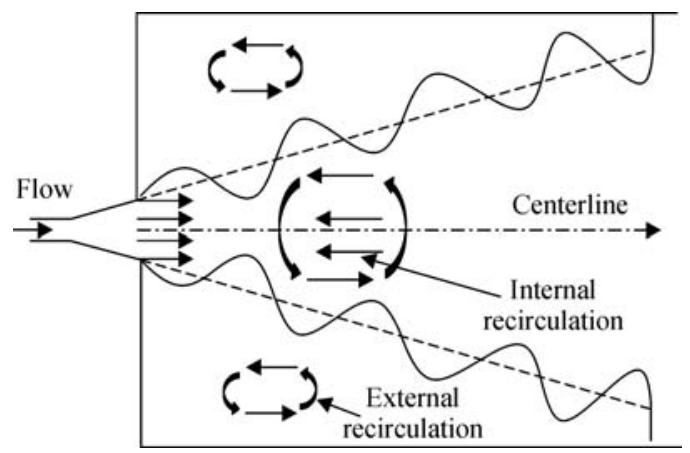

Fig. 10 Schematic illustrating flow recirculation generated by a free jet from nozzle

Fig. 12(b). The maximum impingement force, $F_{\mathrm{im}}$, on the back plate exerted by the gas is calculated from:

$$
F_{\mathrm{im}}=\frac{\pi}{2} \rho v_{\mathrm{im}}^{2} l_{\mathrm{im}}^{2} .
$$

The maximum impact force is about $0.2 \mathrm{~N}$ for an inlet $\mathrm{N}_{2}$ flow of $0.6 \mathrm{~m}^{3} \cdot \mathrm{h}^{-1}$. The impingement impact by the jet flow on the back plate is insignificant. It can be assumed that the modified nozzle design can offer promising flow characteristics.

\subsubsection{Temperature profile}

The heat transfer characteristics inside the solar receiver was analyzed numerically by applying the boundary conditions described in Section 4.2. Figure 13 shows the contours for the static temperature in mid-plane along the length of the receiver. The temperature distribution shows almost symmetry with respect to the axis planes. It also indicates a promising result as the temperature distribution is nearly uniform along the receiver length.

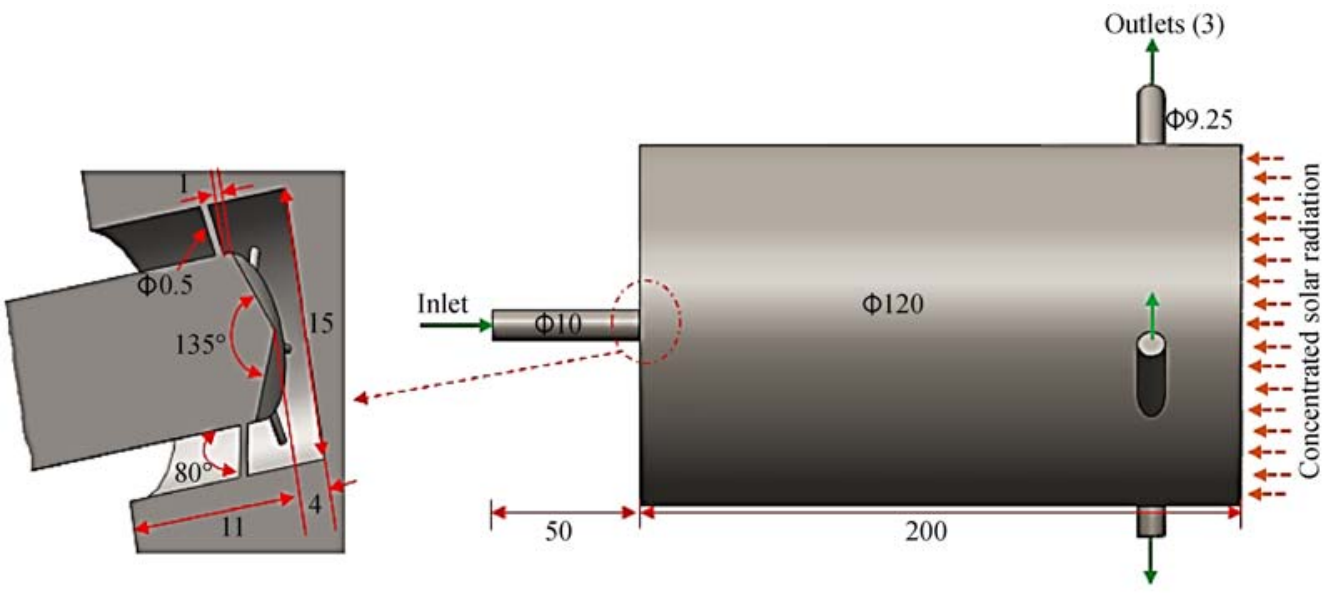

Fig. 11 Receiver geometry with modified nozzle configuration (all dimensions are in $\mathrm{mm}$ )

(a)

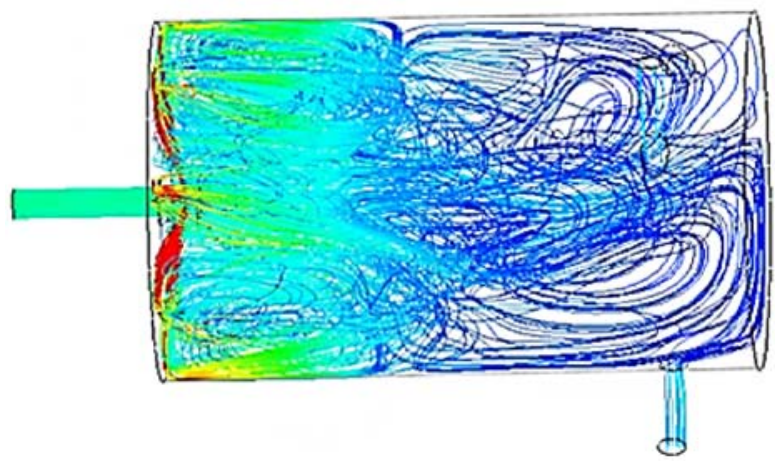

मำ (b)

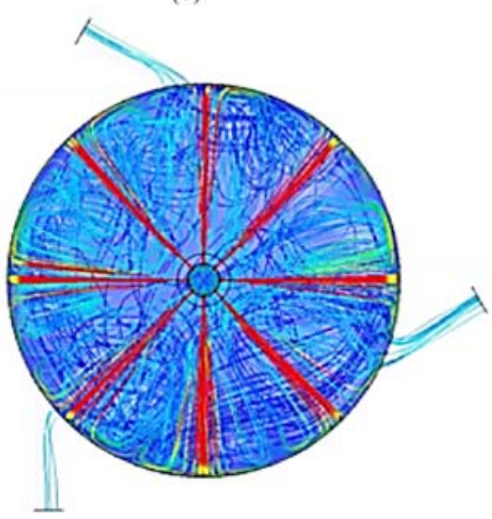

Velocity $/\left(\mathrm{m} \cdot \mathrm{s}^{-1}\right)$

Fig. 12 Gas velocity streamline: (a) inside the receiver, and (b) at the back plate 
(a)

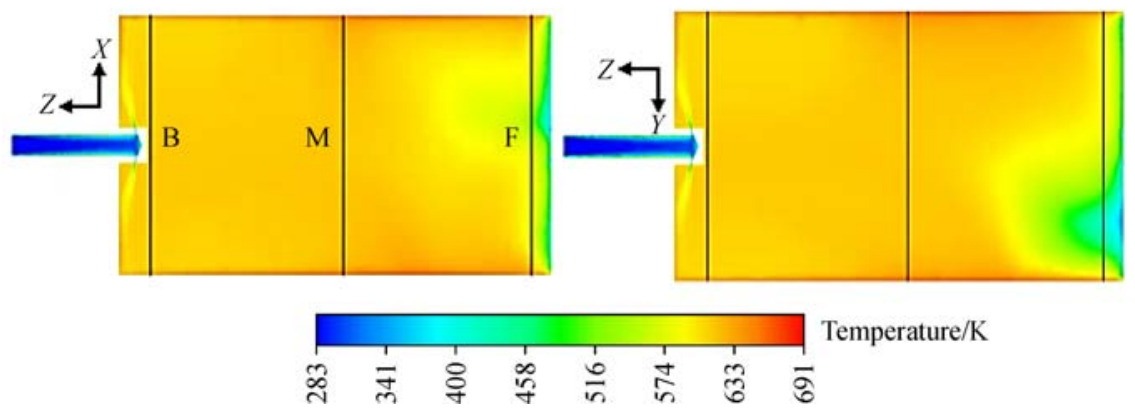

Fig. 13 Contours of static temperature for the modified nozzle along (a) ZX and (b) YZ plane

A centerline temperature distribution is also analyzed to understand the flow dynamics on temperature distribution inside the solar receiver. Figure 14 presents the centerline temperature distribution for three simulations.

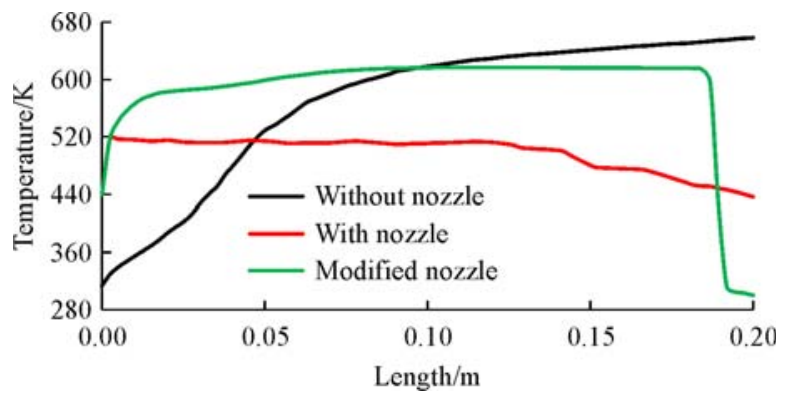

Fig. 14 Centerline static temperature profiles

For the reference case without nozzle, centerline temperature increases with distance, illustrating a large gradient along the centerline. Centerline temperature for nozzles, both previous and modified case, show nearly uniform temperature distribution. The modified nozzle design, thus, shows many promising advantages including a high degree of gas mixing, an increased heat transfer rate, and a uniform temperature distribution along the length of the solar receiver. For any reactive solar thermal process, such improvement in the gas phase mixing and the temperature distribution will enhance the overall performance of the solar thermochemical processes.

\section{Conclusions}

In present study, a jet-type nozzle was adapted from a combustion nozzle design. The nozzle was implemented with an existing solar receiver for the gas injection. Experiments were performed to highlight the applicability of the combustion nozzle concept into the solar receiver design. Experimental result without nozzle showed a clear temperature gradient along the length of the solar receiver, having a maximum of $200 \mathrm{~K}$ temperature difference between the front and back. However, inclusion of the nozzle for the gas injection offered an improvement in the temperature distribution inside the solar receiver, reducing the temperature difference by $41 \mathrm{~K}$. To analysis the flow characteristics and heat transfer inside the solar receiver, CFD simulation was performed and validated against the experimental results. Numerical analysis showed that the gas flow dynamics plays an important role in heat transfer and the corresponding temperature distribution inside the solar receiver. Based on the CFD analysis, a modification of the nozzle was proposed. Adapting such a modification showed very promising results on gas circulation and mixing and heat transfer. Numerical results show an encouraging insight on uniform gas temperature distribution inside the solar receiver. This observation also highlights a potential alternative to counter the hotspot issue. However, experimental validation is required to support this claim which is the scope of future work.

Acknowledgements Experimental portion of this research was funded by the Impulse Fund project \# IMP/14/049 of KU Leuven. The authors also acknowledge the Minnesota Supercomputing Institute (MSI) at the University of Minnesota for providing resources that contributed to the research results reported within this paper.

\section{Nomenclature}

$a=$ absorption coefficient $/ \mathrm{m}^{-1}$

$C_{1 \varepsilon}, C_{2 \varepsilon}, C_{\mu}, \alpha_{\varepsilon}, \alpha_{\mathrm{k},} A_{0}, A_{\mathrm{s}}, \eta, \gamma, W, U^{*}=$ realizable RNG $k-$ $\varepsilon$ model parameters

$C_{\mathrm{p}}=$ specific heat $/\left(\mathrm{J} \cdot \mathrm{mol}^{-1} \cdot \mathrm{K}^{-1}\right)$

$G_{\mathrm{k},} G_{\mathrm{b}}=$ production of turbulent kinetic and buoyancy energy by velocity gradient $/\left(\mathrm{J} \cdot \mathrm{m}^{-3} \cdot \mathrm{s}^{-1}\right)$

$I=$ radiative intensity $/\left(\mathrm{W} \cdot \mathrm{m}^{-2}\right)$

$k=$ turbulent kinetic energy per unit mass $/\left(\mathrm{m}^{2} \cdot \mathrm{s}^{-2}\right)$

$n=$ refractive index

$\vec{r}=$ position vector

$\vec{s}=$ direction vector

$P r_{\mathrm{t}}=$ turbulent Prandtl number

$T=$ temperature $/ \mathrm{K}$

$u_{i}=$ velocity component in $i$ th direction $/\left(\mathrm{m} \cdot \mathrm{s}^{-1}\right)$ 
Greek symbols

$\sigma=$ Stefan Boltzmann constant, $5.67 \times 10^{-8} /\left(\mathrm{W} \cdot \mathrm{m}^{-2} \cdot \mathrm{K}^{-4}\right)$

$\rho=\operatorname{density} /\left(\mathrm{kg} \cdot \mathrm{m}^{-3}\right)$

$\mu_{\mathrm{t}}=$ turbulent viscosity $/\left(\mathrm{kg} \cdot \mathrm{m}^{-1} \cdot \mathrm{s}^{-1}\right)$

$\mu_{\text {eff }}=$ effective viscosity $/\left(\mathrm{kg} \cdot \mathrm{m}^{-1} \cdot \mathrm{s}^{-1}\right)$

$\beta=$ scattering coefficient $/ \mathrm{m}^{-1}$

$\varepsilon=$ dissipation rate of turbulent kinetic energy per unit $\operatorname{mass} /\left(\mathrm{m}^{2} \cdot \mathrm{s}^{-2}\right)$

Subscripts

eff $=$ effective

$g=$ gas

$s s=$ stainless steel

ins $=$ insulation

$q u a=$ quartz

\section{References}

1. Yadav D, Banerjee R. A review of solar thermochemical processes. Renewable \& Sustainable Energy Reviews, 2016, 54: 497-532

2. Alonso E, Romero M. Review of experimental investigation on directly irradiated particles solar reactors. Renewable \& Sustainable Energy Reviews, 2015, 41: 53-67

3. Villafán-Vidales H, Arancibia-Bulnes C, Riveros-Rosas D, RomeroParedes H, Estrada C. An overview of the solar thermochemical processes for hydrogen and syngas production: Reactors, and facilities. Renewable \& Sustainable Energy Reviews, 2017, 75: 894-908

4. Kogan M, Kogan A. Production of hydrogen and carbon by solar thermal methane splitting. I. The unseeded reactor. International Journal of Hydrogen Energy, 2003, 28(11): 1187-1198

5. Ozalp N, Kanjirakat A. Lagrangian characterization of multi-phase turbulent flow in a solar reactor for particle deposition prediction. International Journal of Hydrogen Energy, 2010, 35(10): 44964507

6. Hirsch D, Steinfeld A. Solar hydrogen production by thermal decomposition of natural gas using a vortex-flow reactor. International Journal of Hydrogen Energy, 2004, 29(1): 47-55

7. Ozalp N, JayaKrishna D. CFD analysis on the influence of helical carving in a vortex flow solar reactor. International Journal of Hydrogen Energy, 2010, 35(12): 6248-6260

8. Abanades S, Kimura H, Otsuka H. Hydrogen production from thermo-catalytic decomposition of methane using carbon black catalysts in an indirectly-irradiated tubular packed-bed solar reactor. International Journal of Hydrogen Energy, 2014, 39(33): 1877018783

9. Kodama T, Gokon N, Cho H S, Matsubara K, Etori T, Takeuchi A, Yokota S, Ito S. Particles fluidized bed receiver/reactor with a beamdown solar concentrating optics: 30-kWth performance test using a big sun-simulator. In: Proceedings of AIP SolarPACES 2015. Cape Town: AIP Publishing, 2016, 120004

10. Kodama T, Enomoto S I, Hatamachi T, Gokon N. Application of an internally circulating fluidized bed for windowed solar chemical reactor with direct irradiation of reacting particles. Journal of Solar Energy Engineering, 2008, 130(1): 014504
11. Roeb M, Sattler C, Klüser R, Monnerie N, de Oliveira L, Konstandopoulos A G, Agrafiotis C, Zaspalis V, Nalbandian L, Steele A, Stobbe P. Solar hydrogen production by a two-step cycle based on mixed iron oxides. Journal of Solar Energy Engineering, 2006, 128(2): 125-133

12. Muhich C L, Ehrhart B D, Al-Shankiti I, Ward B J, Musgrave C B, Weimer A W. A review and perspective of efficient hydrogen generation via solar thermal water splitting. Wiley Interdisciplinary Reviews. Energy and Environment, 2016, 5(3): 261-287

13. Bertocchi R, Karni J, Kribus A. Experimental evaluation of a nonisothermal high temperature solar particle receiver. Energy, 2004, 29 (5): 687-700

14. Kodama T, Bellan S, Gokon N, Cho H S. Particle reactors for solar thermochemical processes. Solar Energy, 2017, 156: 113-132

15. Levêque G, Bader R, Lipiński W, Haussener S. High-flux optical systems for solar thermochemistry. Solar Energy, 2017, 156: 133148

16. Ophoff C, Abedini-Najafabadi H, Bogaerts J, Ozalp N, Moens D. An Overview of variable aperture mechanism in attempt to control temperature inside solar cavity receivers. In: Proceedings of ASTFE TFEC-2018. Fort Lauderdale: ASTFE, 2018, 747-758

17. Costandy J, Ghazal N, Mohamed M T, Menon A, Shilapuram V, Ozalp N. Effect of reactor geometry on the temperature distribution of hydrogen producing solar reactors. International Journal of Hydrogen Energy, 2012, 37(21): 16581-16590

18. Klein H H, Rubin R, Karni J. Experimental evaluation of particle consumption in a particle seeded solar receiver. Journal of Solar Energy Engineering, 2008, 130(1): 011012

19. Rodat S, Abanades S, Flamant G. Co-production of hydrogen and carbon black from solar thermal methane splitting in a tubular reactor prototype. Solar Energy, 2011, 85(4): 645-652

20. Koepf E, Villasmil W, Meier A. Pilot-scale solar reactor operation and characterization for fuel production via the $\mathrm{Zn} / \mathrm{ZnO}$ thermochemical cycle. Applied Energy, 2016, 165: 1004-1023

21. Mullinger P, Jenkins B. Industrial and process furnaces: Principles, design and operation. Oxford: Butterworth-Heinemann, 2008, 4757

22. Spalding D B. Combustion and Mass Transfer. Oxford: Pergamon Press, 1979, 199-217

23. Abedini-Najafabadi H, Ozalp N. Development of a control model to regulate temperature in a solar receiver. Renewable Energy, 2017, 111: 95-104

24. Vanierschot M. Fluid mechanics and control of annular jets with and without swirl. Dissertation for the Doctoral Degree. Leuven: Katholieke Universiteit Leuven, 2007, 15-20

25. Heylen J. Design, manufacturing and experimental testing of a novel solar reactor. Dissertation for the Masters Degree. Leuven: Katholieke Universiteit Leuven, 2026, 17-18

26. Chien M H, Ozalp N, Morrison G. Computational fluid dynamics and heat transfer analysis of vortex formation in a solar reactor. Journal of Thermal Science and Engineering Applications, 2015, 7 (4): 041007-041008

27. Chase M W. NIST-JANAF Thermochemical Tables: Monograph 9. Maryland: NIST, 1998, 1062

28. Yang W C. Handbook of Fluidization and Fluid-particle Systems. 
New York: CRC press, 2003, 13-15

29. Plawsky J L. Transport phenomena fundamentals. New York: CRC press, 2014, 919-938

30. Fries T, Omerović S, Schöllhammer D, Steidl J. Higher-order meshing of implicit geometries - part I: Integration and interpolation in cut elements. Computer Methods in Applied Mechanics and Engineering, 2017, 313: 759-784

31. Levêque G, Abanades S. Design and operation of a solar-driven thermogravimeter for high temperature kinetic analysis of solid-gas thermochemical reactions in controlled atmosphere. Solar Energy, 2014, 105: 225-235

32. Karabay H, Wilson M, Owen J M. Predictions of effect of swirl on flow and heat transfer in a rotating cavity. International Journal of Heat and Fluid Flow, 2001, 22(2): 143-155

33. Witze P O. Centerline velocity decay of compressible free jets. AIAA Journal, 1974, 12(4): 417-418

34. Ball C, Fellouah H, Pollard A. The flow field in turbulent round free jets. Progress in Aerospace Sciences, 2012, 50: 1-26

35. Dhotre M, Joshi J. Design of a gas distributor: Three-dimensional CFD simulation of a coupled system consisting of a gas chamber and a bubble column. Chemical Engineering Journal, 2007, 125(3): 149-163

36. Chan C, Lam K. Centerline velocity decay of a circular jet in a counterflowing stream. Physics of Fluids, 1998, 10(3): 637-644 